\title{
Patient nonadherence to medication in inflammatory bowel disease
}

\author{
Alan BR Thomson MD PhD FRCPC FACP
}

\begin{abstract}
ARTICLE
Sewitch MJ, Abrahamowicz M, Barkun A, Bitton A, Wild GE, Cohen A, Dobkin PL. Patient nonadherence to medication in inflammatory bowel disease. Am J Gastroenterol 2003;98:1535-44.
\end{abstract}

\section{COMMENTARY}

Dr Sewitch and colleagues at the University of Montreal and McGill University undertook a prospective study that provided evidence for the important relationship between patient-physician discordance and patient nonadherence with medication for inflammatory bowel disease (IBD).

There are various theories of adherence (the term "compliance" is no longer politically correct!). The authors' analyses support the communication theory of adherence, which purports that adherence depends on an effective patient-physician dialogue. "Patient-physician discordance" is defined as the difference between patient and physician evaluations of health-related information. It can be measured with the Patient-Physician Discordance Scale, which compares responses by patients and physicians who, immediately following an office encounter, independently complete a questionnaire. Three domains are considered: symptoms and treatment, well-being, and communication and satisfaction. Another important factor in the adherence process is the presence or absence of psychological distress in the patient; indeed, if there is discordance and distress, it may be useful for the physician to schedule follow-up appointments.

For all patients, a low risk of nonadherence was associated with longer duration of IBD, greater belief by the patient that the medication would be beneficial and lower total discordance. These data suggest that patients with more experience with IBD were less likely to deliberately stop taking medication.
Sometimes patients do not adhere to the treatment regimen for unintentional reasons. As Maida Sewitch and her colleagues point out, "a new model of patient adherence has been proposed in which effective patient-physician dialogue is central to promoting patient adherence". Psychosocial factors may contribute not only to patient nonadherence but also to physicians' and patients' healthrelated perceptions. These findings are also in keeping with the health psychology literature, which emphasizes the importance of stress and social support on the adoption of lifestyle changes.

What are the implications of this paper for the practicing gastroenterologist? First, to recognize that "adherence" (or "compliance") is a problem, especially in patients with chronic diseases like Crohn's disease and ulcerative colitis. Secondly, to recognize that nonadherence may be both intentional and unintentional: of the $41 \%$ of the patients who were nonadherent to medication, $81 \%$ indicated that this was accidental. Finally, this paper highlights what we have all suspected for some time "...the therapeutic relationship, as well as individual clinical and psychosocial characteristics, influence adherence to medication".

You probably won't remember the results of the "simple bivariate generalized estimating equations analyses" that "active disease, longer duration of disease, scheduling another appointment, consulting another health professional, prescribed steroids, and higher discordance on well-being were associated with decreased risks of overall nonadherence to medication". What I will remember, however, is that there are:

- Sophisticated measures to predict who will and who will not be adherent;

- Ways of assessing discordance between the patient and physician; and

- Sophisticated psychosocial measures that can be used to evaluate, for example, patient stress. 
Finally, it is important to develop as good a relationship with patients as possible, not just because it is a good thing to do, but also because it may ensure that patients take their medications!

Dr Alan BR Thomson MD PhD FRCPC Division of Gastroenterology, Department of Medicine, University of Alberta, Edmonton, Alberta

The authors respond:

We thank Dr Thomson for such encouraging comments. We agree with him that while description of complex methodology may not be the most attractive part of a clinical research article, it is important in that it allows us to obtain new insights into complex processes including patients' characteristics, patient-physician discordance, and adherence to treatment.

Maida J Sewitch PhD

Division of Clinical Epidemiology, Montreal General Hospital and Department of Medicine, McGill University, Montreal, Quebec

Patricia L Dobkin PhD

Division of Clinical Epidemiology, Montreal General Hospital and

Department of Medicine, McGill University, Montreal, Quebec

Michal Abrahamowicz PhD

Division of Clinical Epidemiology, Montreal General Hospital and Department of Epidemiology, Biostatistics and Occupational Health, McGill University, Montreal, Canada 


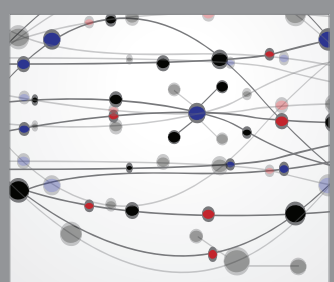

The Scientific World Journal
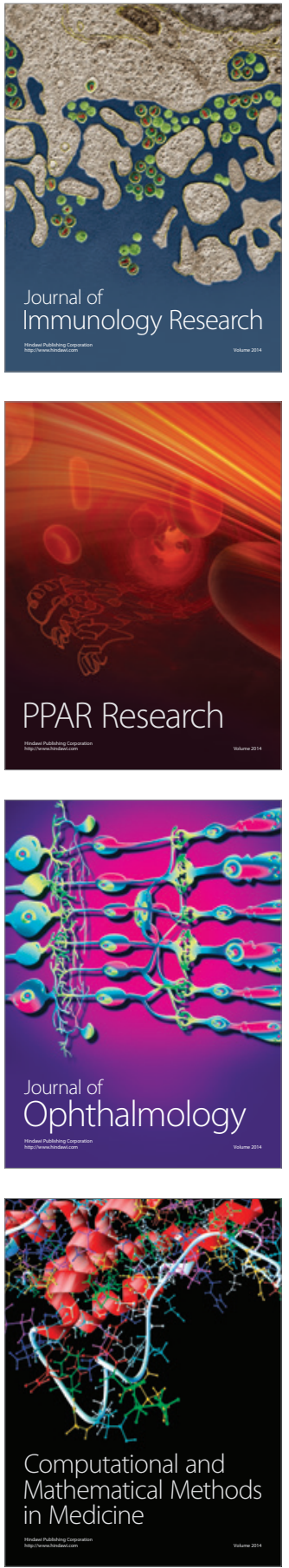

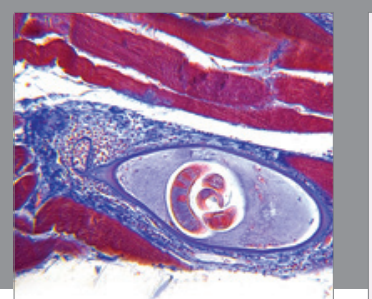

Gastroenterology Research and Practice

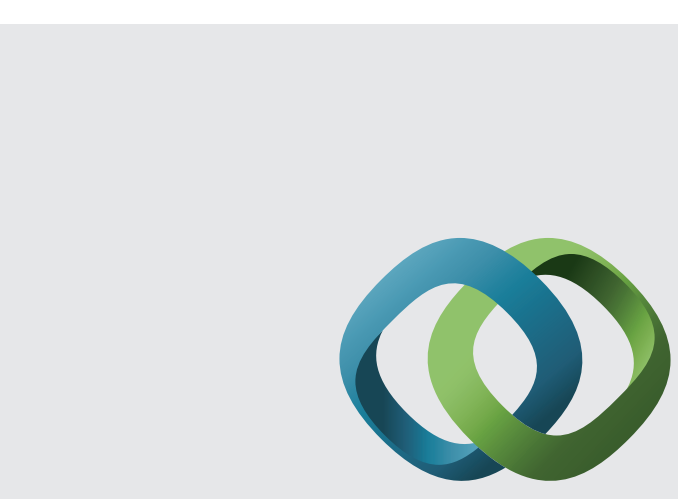

\section{Hindawi}

Submit your manuscripts at

http://www.hindawi.com
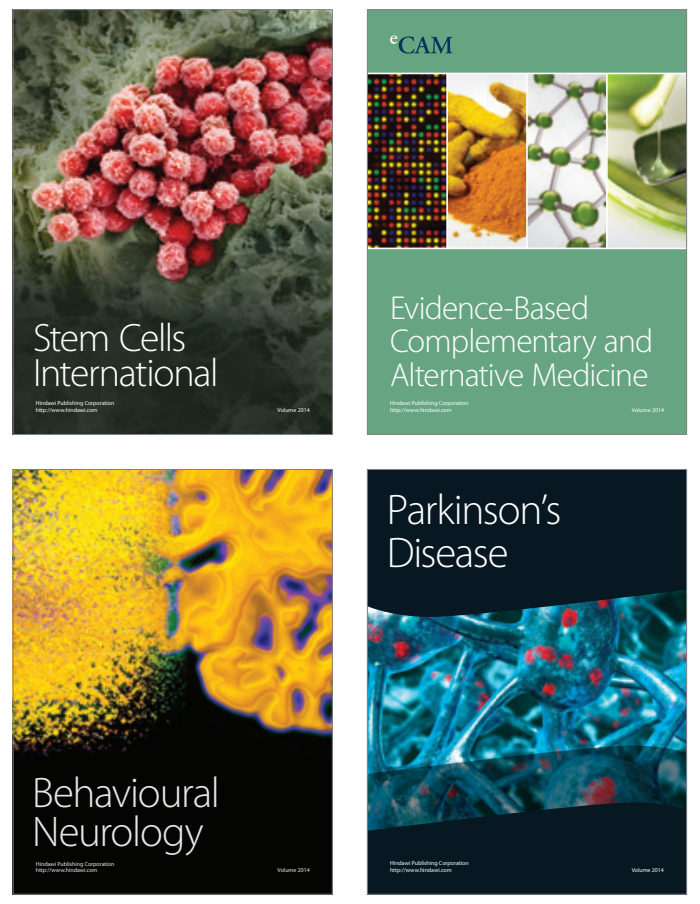
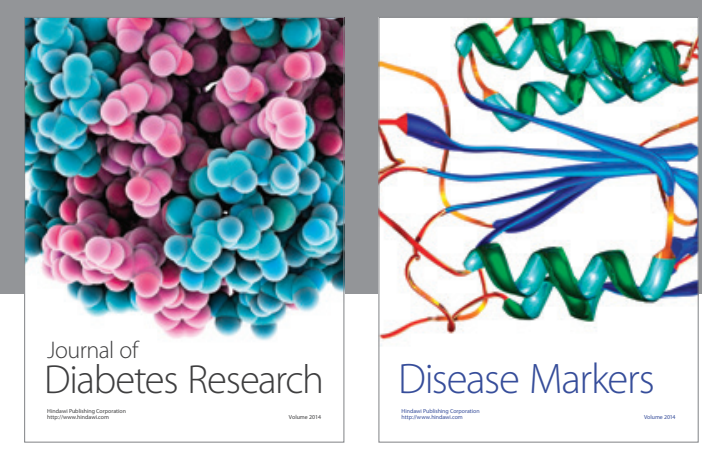

Disease Markers
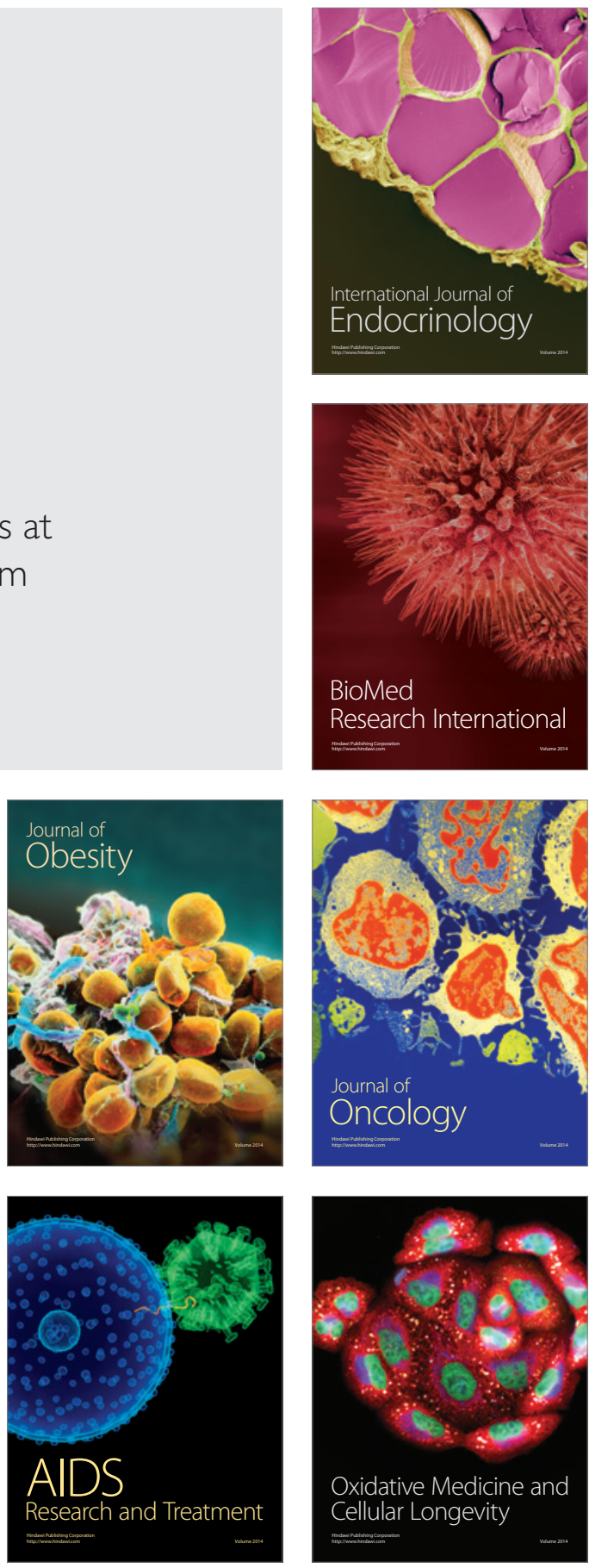